\title{
Comparative acetylome analysis reveals the potential roles of lysine acetylation for DON biosynthesis in Fusarium graminearum
}

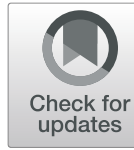

\author{
Shanyue Zhou ${ }^{1 *}$ (D) and Chunlan $\mathrm{Wu}^{2}$
}

\begin{abstract}
Background: Fusarium graminearum is a destructive fungal pathogen of wheat, barley and other small grain cereals. During plant infection, the pathogen produces trichothecene mycotoxin deoxynivalenol (DON), which is harmful to human and livestock. FgGCN5 encodes a GCN5 acetyltransferase. The gene deletion mutant Fggcn5 failed to produce DON. We assumed that lysine acetylation might play a key regulatory role in DON biosynthesis in the fungus.

Results: In this study, the acetylome comparison between Fggcn5 mutant and wild-type strain PH-1 was performed by using affinity enrichment and high resolution LC-MS/MS analysis. Totally, 1875 acetylated proteins were identified in Fggcn5 mutant and PH-1. Among them, 224 and 267 acetylated proteins were identified exclusively in Fggcn5 mutant and $\mathrm{PH}-1$, respectively. Moreover, 95 differentially acetylated proteins were detected at a significantly different level in the gene deletion mutant:43 were up-regulated and 52 were down-regulated. GO enrichment and KEGG-pathways enrichment analyses revealed that acetylation plays a key role in metabolism process in F. graminearum.

Conclusions: Seeing that the gens playing critical roles in DON biosynthesis either in Fggcn5 mutant or PH-1. Therefore, we can draw the conclusion that the regulatory roles of lysine acetylation in DON biosynthesis in $F$. graminearum results from the positive and negative regulation of the related genes. The study would be a foundation to insight into the regulatory mechanism of lysine acetylation on DON biosynthesis.
\end{abstract}

Keywords: Fusarium graminearum, Deoxynivalenol, Lysine acetylation, Acetylome

\section{Background}

Fusarium graminearum is a disastrous fungal pathogen which causes Fusarium head blight (FHB) on wheat, barley and other small grain cereals $[1,2]$. In addition to the severe yield loss and quality damage, the pathogen produces trichothecene-type mycotoxins, such as deoxynivalenol (DON) in the infected tissue. DON is a secondary metabolite, which contributes to the spread of the fungus in the spikelet and contaminates cereal grains and cereal-based products, resulting in a threat to the health of human and livestock [3, 4].

Lysine acetylation is a conserved post-translational modification (PTM) of proteins occurring both in

\footnotetext{
* Correspondence: zhoushanyao@qau.edu.cn

${ }^{1}$ College of Plant Health and Medicine, The Key Lab of Integrated Crop Pests Management of Shandong Province, Qingdao Agricultural University, No. 700 Changcheng Road, Chengyang, Qingdao 266109, Shandong, China Full list of author information is available at the end of the article
}

eukaryotes and prokaryotes. The modification consists of two reversible reactions: the acetylation, in which the acetyl-groups were added to the lysine residues of target protein by lysine acetyltransferase (KAT); in contrast, the deacetylation is a reversed process to remove the acetyl-groups from the acetylated proteins by lysine deacetylase (KDAC) [5, 6]. The balance of acetylation/deacetylation status of proteins is dynamically regulated by KATs and KDACs in order to achieve their proper roles during numerous cellular processes such as cell morphology, metabolic pathways, protein synthesis [7-9]. The acetylation was first identified in histone proteins, whose acetylated form is responsible for the structure remodeling of the chromatin and activation of genes expression $[10,11]$. In recent years, the protein acetylation has been widely studied by using advanced mass spectrometry based proteomics tool. Global analyses of acetylome have been successfully performed in plants $[12,13]$,

(c) The Author(s). 2019 Open Access This article is distributed under the terms of the Creative Commons Attribution 4.0 International License (http://creativecommons.org/licenses/by/4.0/), which permits unrestricted use, distribution, and reproduction in any medium, provided you give appropriate credit to the original author(s) and the source, provide a link to the Creative Commons license, and indicate if changes were made. The Creative Commons Public Domain Dedication waiver (http://creativecommons.org/publicdomain/zero/1.0/) applies to the data made available in this article, unless otherwise stated. 
fungi $[14,15]$, and prokaryotes [16, 17], revealing that acetylation contributes to diverse protein functions in living cells, including protein localization, enzymatic activity, protein-protein and protein-nucleic acids interaction [18-20].

The lysine acetylation also plays a crucial role in regulating central metabolism as the extensively acetylated enzymes responsible for metabolism have been found in both eukaryotes and prokaryotes [9, 17, 21]. For instance, most enzymes involved in glycolysis, the tricarboxylic (TCA) cycle, gluconeogenesis, the urea cycle, and fatty acid metabolism were acetylated in human liver tissue [22]. A global acetylome analysis in Salmonella enterica revealed that about $90 \%$ of the enzymes of central metabolism were found to be acetylated [8]. In addition, the protein acetylation is also involved in the secondary metabolism process, such as nonribosomal peptide synthesis, hydroxamate siderophore and phosphinic acid products biosynthesis [20].

The gene FgGCN5 (FGRAMPH1_01T00753) in F. graminearum PH-1 encodes a GCN5 acetyltransferase. The most attractive defect of the gene deletion mutant is the functional block in DON biosynthesis [23], indicating that the gene plays a crucial role in producing DON in the fungus. To reveal the potential roles of lysine acetylation in DON biosynthesis, we performed a global acetylome comparison between the gene deletion mutant Fggcn5 and the wild-type strain PH-1. Totally, 2626 acetylated lysine sites in 1875 proteins were identified in Fggcn5 mutant and PH-1.

\section{Results and discussion}

Difference of the acetylated proteins between the wild type and Fggen5 deletion mutant

The predicted gene in the $F$. graminearum genome, FGRAMPH1_01T00753, is orthologous to yeast GCN5 and its lysine acetyltransferase activity was confirmed in a previous study [23]. To gain insights into the possible acetylome regulated by $F g G C N 5$ in F. graminearum, we generated the gene deletion construct by the split- marker approach [24] and transformed it into the wildtype strain PH-1. As shown in Fig. 1, the Fggcn5 deletion mutant significantly reduced hyphae growth (growth rate is $53.45 \%$ of $\mathrm{PH}-1$ ), and failed to produce DON.

To identify proteins acetylated by FgGCN5, total proteins were isolated from PH-1 and Fggcn5 mutant. After digestion with trypsin, lysine-acetylated peptides were enriched with the anti-acetyl-lysine antibody and analyzed with LC-MS/MS as described [25]. A total of 2626 lysine acetylation sites (Additional file 1: Table S1) were identified in 1875 proteins from PH-1 and Fggcn5 mutant (Additional file 2: Table S2). Among them, 95 proteins were differentially acetylated at a significant level of Ratio $>+1-2(p<0.05)$ in the Fggcn5 deletion mutant in comparison with $\mathrm{PH}-1.43$ proteins were upregulated, 52 were down-regulated in the mutant (Additional file 3: Table S3). It is possible that the acetylation down-regulated proteins in the Fggcn5 mutant function in a positive slight role in the DON biosynthesis, while the up-regulated proteins play the opposite role.

In comparison with the Fggcn5 mutant, 274 acetylated lysine sites of the 267 proteins were identified exclusively in the wild-type strain PH-1 (Additional file 4: Table S4). Some proteins likely to be acetylated by FgGCN5 have been functionally characterized (Table 1 ). We also identified 226 acetylated lysine sites in 224 proteins that only present in the Fggcn 5 mutant. Deletion in FgGCN5 somehow stimulated acetylation on these proteins in $F$. graminearum. It is possible that other lysine acetyltransferases were activated to acetylate these proteins in the absence of $F g G C N 5$. Some of these proteins have been functionally characterized (Table 2).

The abundance of acetylated proteins detected in this study indicated that lysine acetylation is a common protein modification in $F$. graminearum similar to the observations in other living organisms [12-15]. Approximately $14.24 \%$ of the acetylated proteins identified in this study were only detected in the wild type strain and are potential targets of $F g G C N 5$ lysine acetyltransferase.
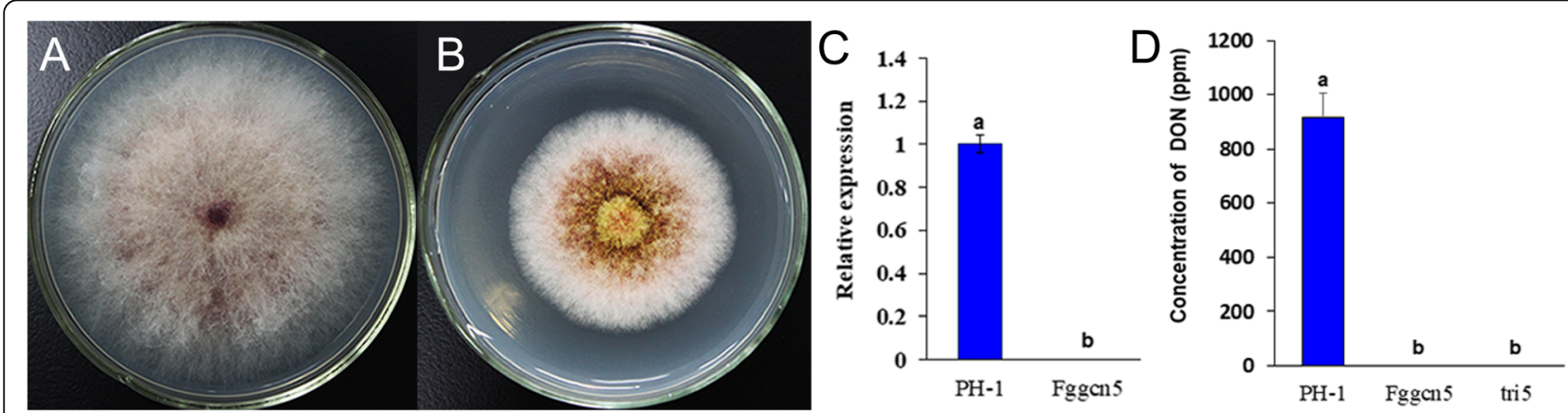

Fig. 1 Colony and DON production in Fggen5 mutant. a Colony of the wild-type strain PH-1. b Colony of the Fggcn5 mutant on PDA. c Expression of the FgGCN5 gene in $\mathrm{PH}-1$ and Fggen5 mutant. $\mathbf{d}$ DON production in Fggcn5 mutant, $\mathrm{PH}-1$ and negative control tri5 mutant 
Table 1 Acetylated proteins specially detected in wild type strain $\mathrm{PH}-1$

\begin{tabular}{lllll}
\hline Protein & Gene number & Annotation & Function & Reference \\
\hline FgFkbp12 & FGSG_09690 & Rapamycin binding protein & Rapamycin toxicity & [26] \\
FaTUA1 & FGSG_00639 & a-tubulin & Virulence, hyphae growth & [27] \\
GzOB031 & FGSG_08737 & Transcription factor & Virulence & [28] \\
GzBrom002 & FGSG_06291 & Transcription factor & DON, virulence, sexual and asexual & [28] \\
FGSG_10825 & FGSG_10825 & Homocysteine transferase & DON, virulence and development & [29] \\
FGK3 & FGSG_07329 & Glycogen synthase kinase & DON, virulence and development & [30] \\
PKR & FGSG_09908 & Protein kinase & DON, virulence, sexual and asexual & [31] \\
FCA6 & FGSG_02974 & Peroxidase & Peroxidase activities & [32] \\
\hline
\end{tabular}

Functional annotation and enrichment analysis of the proteins differentially acetylated in $\mathrm{PH}-1$ and the Fggen5 mutant

To determine the functions of acetylated proteins, we analyzed GO annotation and classified the identified proteins according to their biological processes, molecular functions and cellular compartments. In the GO biological processes, 59 proteins were involved in metabolic processes, 59 in cellular processes, 15 in biological regulation, and 14 in the regulation of biological processes and cellular compartment organization or biogenesis. According to GO molecular function category, 42 proteins were involved in catalytic activities, 45 in binding activities, and 17 in structural molecular activity. With respect to the cellular compartments on level 2, 59 proteins were cell proteins, 58 were cell part proteins, 49 were organelle proteins, 30 were macromolecularcomplex proteins, 24 were organelle part proteins, and 10 were membrane-enclosed lumen proteins (Fig. 2a).

Furthermore, the GO enrichment analysis was performed to identify the biological processes and molecular functions of the acetylated proteins (Fig. 2b, Additional file 5: Table S5). The results showed that the acetylated proteins identified in this study were significantly enriched in several GO biological processes, including monocarboxylic acid metabolism, pyridine nucleotide metabolism, nicotinamide nucleotide metabolism, pyruvate metabolic process, glucose 6-phosphate metabolic process, glyceraldehyde-3- phosphate metabolic process, and NADP metabolic process. In the GO molecular functions, most of the acetylated proteins were significantly enriched in structural molecular activity, structural constituent of ribosome, oxidoreductase activity. From the GO cellular compartment categories, we found that a great proportion of the identified acetylated proteins were in intracellular non-membranebounded organelles, ribonucleoprotein complexes, and ribosome.

The KEGG-pathways in which the acetylated proteins involved were analyzed (Fig. 3a). The results revealed that proteins were enriched in several conserved pathways such as ribosomes, glycolysis/gluconeogenesis and citrate cycle (TCA cycle) (Fig. 3b, Additional file 6: Table S6). Moreover, the KEGG-pathways enriched in fatty acid biosynthesis, pyruvate metabolism suggested that the acetylation play important roles in cell metabolic processes. It is in agreement with the well-established conclusion that lysine acetylation plays key roles in regulation of the metabolic pathways $[8,17,40]$. Further, the acetylate form of the enzymes involved in the acetylCoA synthesis, such as pyruvate dehydrogenase E2 (FG04171.1) and long-chain acyl-CoA synthetase

Table 2 Acetylated proteins specially detected in Fggen5 mutant

\begin{tabular}{llll}
\hline Protein & Gene number & Annotation & Function \\
\hline GzHMG002 & FGSG_00385 & Transcription factor & DON, virulence and development \\
GzCCHC011 & FGSG_10716 & Transcription factor & DON, virulence and development \\
GzZC230 & FGSG_07133 & Transcription factor & DON, virulence \\
FgHXK1 & FGSG_00500 & Hexokinase & DON, virulence and development \\
FgSKN7 & FGSG_06359 & Transcription factor & DON, virulence and development \\
FaMyo2B & FGSG_07469 & Myosin protein & Virulence and development \\
FgArb1 & FGSG_04181 & ABC transporter & DON, virulence and development \\
FgATG8 & FGSG_10740 [34] & Sexual and asexual development \\
CDC2B & FGSG_03132 & Autophagy protein & As5] \\
TRI15 & FGSG_11205 & Kinase & Donal and vegetative growth \\
\hline
\end{tabular}




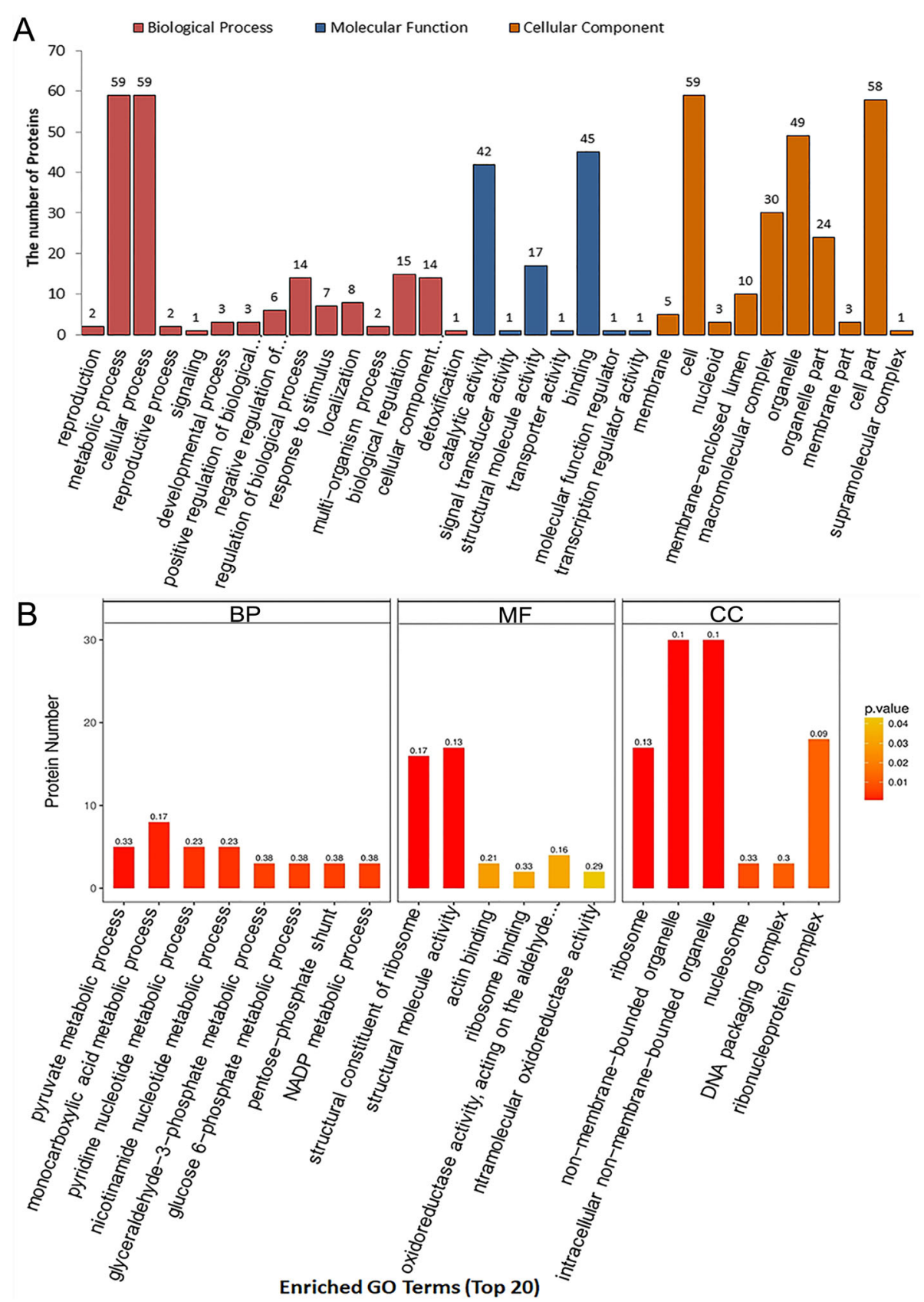

Fig. $2 \mathrm{GO}$ and $\mathrm{GO}$ enrichment of the identified acetylated proteins. a GO analysis of the identified acetylated proteins. The proteins were classified according to their biological processes, molecular functions and cellular compartments. Numbers of proteins in different classification were shown on top of the columns. b GO enrichment analysis of the identified acetylated proteins

(FG08543.1) were detected only in PH-1 but not in the Fggcn 5 mutant. It should be noted that the acetyl-CoA is essential for the DON synthesis. Therefore, the GO and KEGG enrichment provided powerful evidence for the role of acetylation in DON synthesis.

\section{Protein-protein interaction network analysis}

To better understand the cellular processes regulated by lysine acetylation, the protein-protein interaction network was predicted as described [41]. In total, 93 acetylated proteins were mapped into the proteinprotein interaction network (Additional file 7: Table S7). As shown in Fig. 4, the network overviews the physical and functional interactions of the acetylated proteins in F. graminearum. Obviously, the ribosome-associated proteins, metabolism-associated proteins, especially proteins involved in the citrate cycle were specifically enriched. These findings suggest that acetylation plays a 


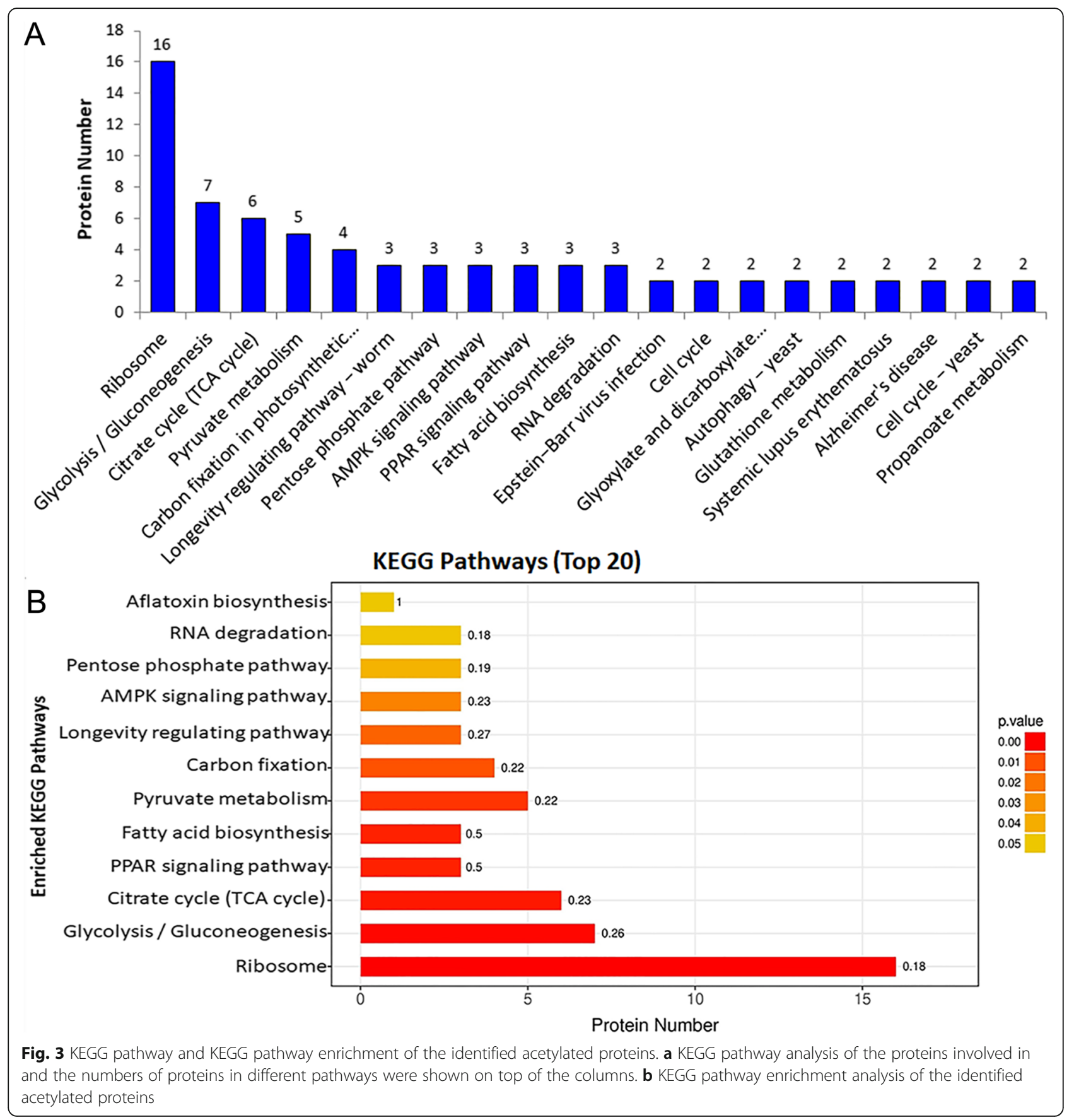

key role in protein biosynthesis and central metabolic processes. Interestingly, the core component of nucleosome Histone $\mathrm{H} 3$ and Histone $\mathrm{H} 2 \mathrm{~B}$ were involved in the network. The H3-interacting protein (FGSG-08173) is predicted to be homologous to the Pim1 Ser/Thr protein kinase, which plays an important role in signal transduction related to energy metabolism and cell proliferation and survival in humans [42, 43]. Another H3-interacting protein (FGSG_10,040) is predicted to encode FACT complex subunit SPT16, which was demonstrated to participate in specific regulation on genes transcription in yeast [44]. It was well demonstrated that acetylation of histone $\mathrm{H} 3$ by FgGCN5 is directly related to DON biosynthesis [45]. It is possible that histone $\mathrm{H} 3$ was coregulated by FgGCN5 and kinase FGSG-08173. The acetylation and phosphorylation of histone $\mathrm{H} 3$ contributes to the activation or inactivation of FGSG-10040 in the FACT complex, which in turn affects the re- 


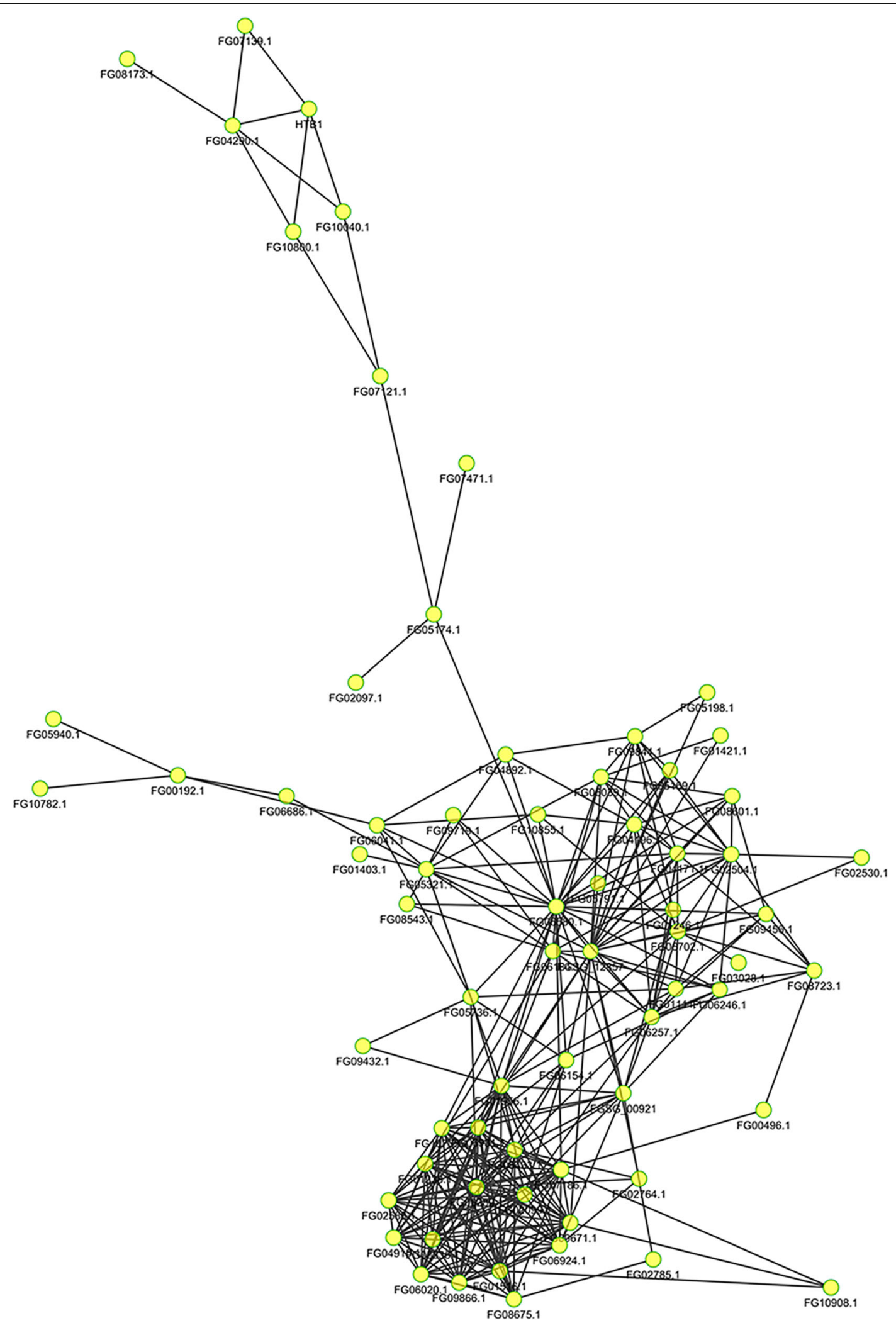

Fig. 4 Protein-protein interaction network of the acetylated proteins. 93 acetylated proteins were mapped into the protein-protein interaction network using STRING database 
organization of nucleosomes. As a result, the transcription of genes involved in the DON production were initiated or blocked.

Proteins acetylated in PH-1 involved in DON biosynthesis Since the Fggcn5 gene deletion mutant was defective in DON biosynthesis, lysine acetylation manipulated by FgGCN5 likely plays important regulatory roles in DON biosynthesis in F. graminearum. In this study, we found that some proteins involved in DON production are the potential acetylation targets of FgGCN5.

GzBrom002 (FGSG_06291) encoding a transcription factor plays essential roles in DON production, virulence, asexual and sexual reproduction. The gene deletion mutant GzBrom002 completely lost virulence on wheat, ability in DON production and asexual and sexual spores production [28]. A Homocysteine transferase gene (FGSG_10825) is also multifunctional in F. graminearum. Phenotype assays showed that the virulence and DON production were reduced in the gene deletion mutant Moreover, the mutant failed to produce perithecia and aerial mycelia [29]. Another gene FGK3 (FGSG07329), encodes a glycogen synthase kinase orthologous to mammalian GSK3. The gene deletion mutant $\Delta f g k 3$ is defective in DON production [30]. FgGCN5 might positively regulate DON biosynthesis through acetylating these proteins.

It has been well demonstrated that cAMP- dependent protein kinase (PKA) plays critical roles in DON biosynthesis in F. graminearum $[46,47]$. In this study, PKR (FGSG_09908), the regulatory subunit of PKA, was found to be acetylated in PH-1 but not in the Fggcn5 mutant. The result indicates that the PKR may be one of the substrates of FgGCN5 acetyltransferase in F. graminearum. However, PKR acts as a negative factor in DON production as the DON content was increased in the gene deletion mutant of PKR [31]. This suggests that the negative effect of PRK on DON biosynthesis may be limited by FgGCN5 through lysine acetylation.

\section{Proteins acetylated in Fggen5 mutant are associated with DON production}

Proteins acetylated specifically in Fggcn5 mutant were identified as well, suggesting that the proteins are targets of other KATs rather than FgGCN5. Interestingly, some proteins were proved to be associated with DON biosynthesis.

It is well known on the functions of some TRI genes in DON biosynthesis. In this study, the acetylated TRI15 (FGSG_11205) was detected only in the Fggcn5 mutant. TRI15, encoding a Cys2-His2 zinc finger protein, acts as a negative regulator of the trichothecene biosynthetic genes $[39,48]$. It is likely that TRI15 is activated by other
KATs and thereby plays a negative role in DON production in Fggcn5 mutant.

Additionally, FgHXK1(FGSG_00500) encodes a ratelimiting enzyme in DON biosynthesis. DON production was severely inhibited in the gene deletion mutant. Moreover, the $\triangle F g H X K 1$ mutant is nonpathogenic on wheat, defective in hyphae growth and conidiation [33]. Some transcription factors identified in this study were also characterized to play key roles in DON production and pathogen virulence including GzHMG002 (FGSG 00385), GzCCHC011 (FGSG_10716) and GzZC230 (FGSG_07133) [28]. Recently, a ATP-binding cassette (ABC) transporte FgArb1 (FGSG_04181) was proved to function in pathogenesis and DON production in F. graminearum, as the virulence and DON production were dramatically reduced in the gene deletion mutant [36]. It is likely that acetylation of these proteins by other KATs in the absence of FgGCN5 leads to the inactivation of the genes, and finally leads to the inhibition of the DON production in Fggcn5 mutant.

\section{Conclusions}

In summary, the acetylome comparison between Fggcn5 mutant and $\mathrm{PH}-1$ was performed by high throughput proteomics analysis. The differentially acetylated proteins were identified. Our results indicate that genes play critical roles in DON production in Fggen5 mutant or $\mathrm{PH}-1$. Therefore, we can draw the conclusion that the DON biosynthesis in F. graminearum was properly regulated by lysine acetylation both in positive and negative ways. The study would be a foundation to insight into the regulatory mechanism of lysine acetylation on DON production.

\section{Methods}

\section{Generation of Fggen5 mutant}

The gene deletion mutant Fggcn5 was generated with the split-marker approach [24]. For the mutant, a $790 \mathrm{bp}$ upstream and an $820 \mathrm{bp}$ downstream flanking DNA sequences of $\mathrm{FgGCN5}$ were amplified with primers 280$1 F / 280-2 R$ and $280-3 F / 280-4 R$, respectively (Table 3 ). The PCR products were then connected to the hygromycin phosphotransferase $(h p h)$ fragments amplified with the primers HYG-F/HY-R and YG-F/HYG-R (Table 3) by overlapping PCR. And the resulting PCR products were then transformed into protoplasts of $\mathrm{PH}-1$ following a described method [49]. For protoplasts production, the conidia of PH-1 were incubated in YEPD (yeast extract $3 \mathrm{~g}$, peptone $10 \mathrm{~g}$, dextrose $20 \mathrm{~g}$ per liter) broth at $25^{\circ} \mathrm{C}$. After incubation for $12 \mathrm{~h}$, the mycelia were harvested by filtration with sterile microcloth and digested in lysing buffer $(25 \mathrm{mg} / \mathrm{mL}$ driselase and $5 \mathrm{mg} / \mathrm{mL}$ lysing enzyme in $1.2 \mathrm{M} \mathrm{KCL}$ ) for $2 \mathrm{~h}$. After filtration through a $30 \mu \mathrm{m}$ Nitex nylon membrane, the digestion mixture 
Table 3 Primers for construction and identification of FgGCN5 gene deletion mutant

\begin{tabular}{ll}
\hline Primer & Sequence \\
\hline $280-1 \mathrm{~F}$ & TAGCGTCTTCTCTTGATTGC \\
$280-1 \mathrm{R}$ & TTGACCTCCACTAGCTCCAGCCAAGCC \\
& ATGATTGGTGCGGCTCAAC \\
$280-2 \mathrm{~F}$ & GAATAGAGTAGATGCCGACCGCGGGTT \\
& AACTAAAAGCGGGAATCGG \\
$280-2 \mathrm{R}$ & ACCAAGACCTATCACAGCAC \\
HYG-F & GGCTTGGCTGGAGCTAGTGGAGGTCAA \\
HY-R & GTATTGACCGATTCCTTGCGGTCCGAA \\
YG-F & GATGTAGGAGGGCGTGGATATGTCCT \\
HYG-R & AACCCGCGGTCGGCATCTACTCTATTC \\
JD280-F & TCGAAGAGCGCAATGGTG \\
JD280-R & TAGCGAATCCGTGGCAAC \\
\hline
\end{tabular}

was centrifugated at room temperature. The protoplasts were gently resuspended in STC buffer (20\% sucrose, 10 $\mathrm{mM}$ tris $\mathrm{pH} 8.0,50 \mathrm{mM} \mathrm{CaCL} 2$ ). The PCR products were added into the protoplast solution and PTC (40\% PEG8000 in STC) was added subsequently. The solution mixture was added into TB3 (3 g Yeast extract, 3 g Casamino acids, $20 \%$ sucrose in $1 \mathrm{l}$ ) containing Hygromycin $\mathrm{B}$ to $300 \mu \mathrm{g} / \mathrm{mL}$ and pour plates. Then the plates were incubated at $25^{\circ} \mathrm{C}$. The transformants with hygromycin resistance was identified by PCR and further confirmed by qRT-PCR with primers JD280-F/JD280-R (Table 3). In qRT-PCR assay, the tub2 was used as an internal control.

\section{DON content measurement assay}

DON content in LTB cultures [50, 51] was assayed with a competitive enzyme-linked immunosorbent assay (ELISA) based DON detection plate kit (Beacon Analytical Systems, Inc., USA) after incubation at $25^{\circ} \mathrm{C}$ for 5 days, as described by Gardiner et al. [1]. In the assay, the Tri5 deletion mutant was used as a negative control.

\section{Strains culture conditions}

The wild-type strain PH-1 and the gene deletion mutant Fggcn5 of F. graminearum were cultured on potato dextrose agar (PDA) at $25^{\circ} \mathrm{C}$ for $4 \mathrm{~d}$. The mycelia were harvested and ground with MiniBead Beater-16 (Biospec, USA) at $30 \mathrm{~Hz}$ for $30 \mathrm{~s}$. The ground mycelium were then cultured in $100 \mathrm{~mL}$ liquid YEPD medium with shaking at $25^{\circ} \mathrm{C}$ for $24 \mathrm{~h}$. Subsequently, the mycelium was collected by filtration with sterile macrocloth and was then incubated in DON inducing medium in the dark with shaking at $25^{\circ} \mathrm{C}$ for $4 \mathrm{~d}$. The DON inducing medium was prepared as described by Gardiner et al. [1].

\section{Protein extraction and trypsin digestion}

The procedures of protein extraction and peptide digestion were modified from a previous report [15]. In brief, the mycelia samples were harvested and ground into cell powder in liquid nitrogen. The resulting cell powder was then transferred into $5 \times$ volume of TCA/acetone (1:9, containing $65 \mathrm{mM} \mathrm{DTT}$ ) and mixed by vortex. After placed at $-20{ }^{\circ} \mathrm{C}$ overnight, the mixture was centrifuged at $7000 \times \mathrm{g}$ for $20 \mathrm{~min}$ at $4{ }^{\circ} \mathrm{C}$. The precipitate was washed with ice-cold acetone for three times and was air-dried at $4{ }^{\circ} \mathrm{C}$. The dried precipitate was then resolved in UA buffer ( $8 \mathrm{M}$ urea, $150 \mathrm{mM}$ Tris- $\mathrm{HCl}, \mathrm{Ph} 8.0$ ) and sonicated 10 times $(10 \mathrm{~s}$ burst with a $15 \mathrm{~s}$ interval for each time) on ice using a high intensity ultrasonic processor (Scientz, Ningbo, China). After centrifuged at $14,000 \mathrm{~g}$ for $40 \mathrm{~min}$, the supernatant was filtered with $0.45 \mu \mathrm{m}$ filters. The filtrate was quantified with the BCA Protein Assay Kit (Bio-Rad, USA).

The resulting protein solution was reduced with 10 $\mathrm{mM}$ DTT for $1 \mathrm{~h}$ at $37^{\circ} \mathrm{C}$ and alkylated with $20 \mathrm{mM}$ iodoacetamide for $45 \mathrm{~min}$ at room temperature in darkness. For trypsin digestion, $100 \mathrm{mM}\left(\mathrm{NH}_{4}\right)_{2} \mathrm{CO}_{3}$ was added to urea concentration less than $2 \mathrm{M}$. Finally, trypsin was added at an enzyme-to-substrate mass ratio of 1 : 50 overnight and additional trypsin was added at an enzyme-to-substrate mass ratio of $1: 100$ for $4 \mathrm{~h}$ to ensure complete digestion.

\section{Immunoaffinity enrichment}

The sample was separated into fractions by high $\mathrm{pH}$ reverse-phase HPLC followed Zhou et al. [15]. As a result, the tryptic peptides were separated into 6 fractions which were then dried by vacuum centrifuging.

The purification and enrichment of lysine acetylated peptides were performed as described $[15,52]$. Briefly, tryptic peptides were re-dissolved in NETN buffer (100 $\mathrm{mM} \mathrm{NaCl}, 1 \mathrm{mM}$ EDTA, $50 \mathrm{mM}$ Tris- $\mathrm{HCl}, 0.5 \% \mathrm{NP}-40$, $\mathrm{pH}$ 8.0). Subsequently, the pre-washed agaroseconjugated anti-acetyllysine antibody beads (Cat. No. 104, PTM Biolabs, Hangzhou, China) were added. The tryptic peptides were incubated at $4{ }^{\circ} \mathrm{C}$ overnight with gentle shaking. The beads were then washed four times with NETN buffer and twice with pure water. Finally, the bound peptides were eluted from the beads with $0.1 \%$ trifluoroacetic acid and were then vacuum-dried. The resulting peptides were cleaned with C18 ZipTips (Millipore, Billerica, MA) according to the manufacturer's instructions before LC-MS/MS analysis.

\section{LC-MS/MS analysis}

LC-MS/MS analysis was performed as described [53] with modification. In the assay, a Q-Exactive mass spectrometer (Thermo Scientific) coupled to Easy nLC (Proxeon Biosystems, now Thermo Fisher Scientific) was 
used. The mass spectrometer was operated in positive ion mode. MS data was acquired using a data-dependent top10 method dynamically choosing the most abundant precursor ions from the survey scan $(300-1800 \mathrm{~m} / \mathrm{z})$ for HCD fragmentation. Automatic gain control (AGC) target was set to $3 \mathrm{e}^{6}$, and maximum inject time to $10 \mathrm{~ms}$. Dynamic exclusion duration was $40.0 \mathrm{~s}$. Survey scans were acquired at a resolution of 70,000 at $\mathrm{m} / \mathrm{z} 200$ and resolution for $\mathrm{HCD}$ spectra was set to 17,500 at $\mathrm{m} / \mathrm{z}$ 200 , and isolation width was $2 \mathrm{~m} / \mathrm{z}$. Normalized collision energy was $30 \mathrm{eV}$ and the underfill ratio, which specifies the minimum percentage of the target value likely to be reached at maximum fill time, was defined as $0.1 \%$. The instrument was run with peptide recognition mode enabled.

\section{Database search}

The resulting MS/MS data was processed using MaxQuant with integrated Andromeda search engine (v.1.4.1.2). Tandem mass spectra were searched against UniProt_F. graminearum database concatenated with reverse decoy database. Trypsin was specified as the cleavage enzyme, the maximum missing cleavage was set as 4 , and up to 5 modifications and 5 charges were allowed in each peptide. Mass error was set at $10 \mathrm{ppm}$ for precursor ions and $0.02 \mathrm{Da}$ for fragment ions. Carbamidomethylation on cysteine was specified as fixed modification and oxidation on methionine, acetylation on lysine and acetylation on protein $\mathrm{N}$-terminal were specified as variable modifications. False discovery rate (FDR) thresholds for peptide and modification site were specified at 0.01 . Minimum peptide length was set as 7 . All the other parameters in MaxQuant were set to default values. The site localization probability was set as 0.75 .

\section{Acetylated protein annotation}

Gene Ontology (GO) annotation of identified acetylated proteins was derived from the UniProt-GOA database (http://www.ebi.ac.uk/GOA/). Firstly, the identified protein ID was converted to UniProt ID, and then mapped to GO ID by protein ID. The proteins were classified by GO annotation based on three categories: biological process, cellular component and molecular function. The subcellular localization of the protein was predicted with WoLF PSORT (http://wolfpsort.seq.cbrc. $\mathrm{jp} /$ ). Secondary structures of proteins were predicted by NetSurfP [54]. Domain descriptions of identified protein were annotated by InterProScan 5 based on protein sequence alignment, and the InterPro domain database (http://www.ebi.ac.uk/interpro/) was used. Kyoto Encyclopedia of Genes and Genomes (KEGG) database was used to annotate protein pathway [55]. Functional annotation tool of DAVID bioinformatics resources 6.7 was used to identify GO terms, KEGG pathways and protein domains [56].

\section{GO, KEGG pathway, domain and motif enrichment analysis}

A two-tailed Fisher's exact test was performed to examine the enrichment of the protein-acetylated entries against all proteins. Correction for multiple hypothesis testing was carried out using a previously described method [57]. Any term with a corrected $p<0.05$ was considered significant.

\section{Acetylation protein-protein interaction network analysis}

The protein-protein interaction was obtained from the STRING database following the methods [41, 25]. The protein-protein interaction network of the identified acetylated proteins was performed with Cytoscape software (version 3.2.1, www.cytoscape.org).

\section{Statistical analysis}

Significant differences between the mutant and wild type strain were calculated according to the peptide intensity using a one-way analysis of variance with SPSS16.05 version. The $p$ values of 0.05 were considered to be statistically significant.

\section{Supplementary information}

Supplementary information accompanies this paper at https://doi.org/10. 1186/s12864-019-6227-7.

Additional file 1: Table S1. The identified acetylated sites in Fggen5 and $\mathrm{PH}-1$.

Additional file 2: Table S2. The identified acetylated proteins in Fggen5 and $\mathrm{PH}-1$.

Additional file 3: Table S3. differentially acetylated proteins at significantly different level in Fggen5 compared with PH-1.

Additional file 4: Table S4. The identified acetylated proteins specific in Fggen5 or PH-1.

Additional file 5: Table S5. GO-based enrichment of the acetylated proteins.

Additional file 6: Table S6. KEGG pathway enrichment analysis.

Additional file 7: Table S7. Protein-protein interaction network of identified acetylated proteins.

\section{Abbreviations}

ABC: ATP-binding cassette; AGC: Automatic gain control; DON: Deoxynivalenol; DTT: Dithiothreitol; ELISA: Enzyme-linked immunosorbent assay; FDR: False discovery rate; FHB: Fusarium head blight; GO: Gene Ontology; HCD: Higher-energy collisionaldissociation; Hph: Hygromycin phosphotransferase; HPLC: High Performance Liquid Chromatography; KAT: Lysine acetyltransferase; KDAC: Lysine deacetylase; KEGG: Kyoto Encyclopedia of Genes and Genomes; PDA: Potato dextrose agar; PKA: CAMP- dependent protein kinase; PTM: Post-translational modification; TCA: Trichloroacetic acid

\section{Acknowledgements}

Not applicable.

Authors' contributions

SZ designed the work, analyzed data and wrote the manuscript. CW conducted the experiments. Both authors have read and approved the final manuscript. 


\section{Funding}

This study was supported by the open project of the State Key Laboratory of Crop Stress Biology for Arid Areas (CSBAA2016001). The funder has no role in the study.

\section{Availability of data and materials}

All data generated or analyzed during this study are included in this published article and its supplementary information files.

\section{Ethics approval and consent to participate}

Not applicable.

\section{Consent for publication}

Not applicable.

\section{Competing interests}

The authors declare that they have no competing of interests.

\section{Author details}

${ }^{1}$ College of Plant Health and Medicine, The Key Lab of Integrated Crop Pests Management of Shandong Province, Qingdao Agricultural University, No. 700 Changcheng Road, Chengyang, Qingdao 266109, Shandong, China. ${ }^{2}$ State Key Laboratory of Crop Stress Biology for Arid Aeras, Northwest A\&F University, Yangling 712100, Shaanxi, China.

Received: 12 May 2019 Accepted: 25 October 2019

\section{Published online: 12 November 2019}

\section{References}

1. Gardiner DM, Kazan K, Manners JM. Nutrient profiling reveals potent inducers of trichothecene biosynthesis in Fusarium graminearum. Fungal Genet Biol. 2009;46:604-13.

2. Suzuki T, Kim YK, Yoshioka H, Iwahashi Y. Regulation of metabolic products and gene expression in Fusarium asiaticum by agmatine addition. Mycotoxin Res. 2013;29:103-11.

3. Audenaert K, Vanheule A, Hofte M, Haesaert G. Deoxynivalenol: a major player in the multifaceted response of Fusarium to its environment. Toxins. 2014;6:1-19.

4. Wang Q, Chen D, Wu M, Zhu J, Jiang C, Xu JR, Liu H. MFS transporters and GABA metabolism are involved in the self-defense against DON in Fusarium graminearum. Front Plant Sci. 2018;9:438.

5. Kouzarides T. Chromatin modifications and their function. Cell. 2007;128: 693-705.

6. Haery L, Thompson RC, Gilmore TD. Histone acetyltransferases and histone deacetylases in B- and T-cell development, physiology and malignancy. Genes Cancer. 2015;6:184-213.

7. Choudhary C, Kumar C, Gnad F, Nielsen ML, Rehman M, Walther TC, Olsen JV, Mann M. Lysine acetylation targets protein complexes and co-regulates major cellular functions. Sci. 2009;325:834-40.

8. Wang Q, Zhang Y, Yang C, Xiong H, Lin Y, Yao J, Li H, Xie L, Zhao W, Yao Y, Ning ZB, Zeng R, Xiong Y, Guan KL, Zhao S, Zhao GP. Acetylation of metabolic enzymes coordinates carbon source utilization and metabolic flux. Sci. 2010;327:1004-7.

9. Nambi S, Gupta K, Bhattacharyya M, Ramakrishnan P, Ravikumar V, Siddiqui $\mathrm{N}$, Thomas AT, Visweswariah SS. Cyclic AMP-dependent protein lysine acylation in mycobacteria regulates fatty acid and propionate metabolism. J Biol Chem. 2013;288:14114-24.

10. Phillips DM. The presence of acetyl groups of histones. Biochem J. 1963;87: 258-63

11. Allfrey VG, Faulkner R, Mirsky AE. Acetylation and methylation of histones and their possible role in the regulation of RNA synthesis. Proc Natl Acad Sci U S A. 1964;51:786-94.

12. Wu X, Oh MH, Schwarz EM, Larue CT, Sivaguru M, Imai BS, Yau PM, Ort DR, Huber SC. Lysine acetylation is a widespread protein modification for diverse proteins in Arabidopsis. Plant Physiol. 2011;155(4):1769-78.

13. Nallamilli BRR, Edelmann MJ, Zhong X, Tan F, Mujahid H, Zhang J, Nanduri B, Peng Z. Global analysis of lysine acetylation suggests the involvement of protein acetylation in diverse biological processes in rice (Oryza sativa). PLoS One. 2014;9(2):e89283.

14. Henriksen P, Wagner SA, Weinert BT, Sharma S, Bacinskaja G, Rehman M, Juffer AH, Walther TC, Lisby M, Choudhary C. Proteome-wide analysis of lysine acetylation suggests its broad regulatory scope in Saccharomyces cerevisiae. Mol Cell Proteomics. 2012;11:1510-22

15. Zhou S, Yang Q, Yin C, Liu L, Liang W. Systematic analysis of the lysine acetylome in Fusarium graminearum. BMC Genomics. 2016. https://doi.org/ 10.1186/s12864-016-3361-3.

16. Liu F, Yang M, Wang X, Yang S, Gu J, Zhou J, Zhang XE, Deng J, Ge F. Acetylome analysis reveals diverse functions of lysine acetylation in Mycobacterium tuberculosis. Mol Cell Proteomics. 2014;13:3352-66.

17. Kim D, Yu B, Lee Y, Choi S, Kang S, Pan J. The acetylproteome of grampositive model bacterium Bacillus subtilis. Proteomics. 2013;13:1726-36.

18. Arif M, Selvi BR, Kundu TK. Lysine acetylation: the tale of a modification from transcription regulation to metabolism. ChemBioChem. 2010;11:1501-4.

19. Downey M, Johnson JR, Davey NE, Newton BW, Johnson TL, Galaang S, Seller CA, Krogan N, Toczyski DP. Acetylome profiling reveals overlap in the regulation of diverse processes by sirtuins, gcn5, and esa1. Mol Cell Proteomics. 2015;14:162-76.

20. Liao G, Xie L, Li X, Cheng Z, Xie J. Unexpected extensive lysine acetylation in the trump-card antibiotic producer Streptomyces roseosporus revealed by proteome-wide profiling. J Proteome. 2014;106:260-9.

21. Choudhary C, Weinert BT, Nishida Y, Verdin E, Mann M. The growing landscape of lysine acetylation links metabolism and cell signaling. Nat Rev Mol Cell Biol. 2014;15:536-50

22. Zhao $S, X u$ W, Jiang W, Yu W, Lin Y, Zhang T, Yao J, Zhou L, Zeng Y, Li H, Li Y, Shi J, An W, Hancock S, He F, Qin L, Chin J, Yang P, Chen X, Lei Q, Xiong $Y$, Guan KL. Regulation of cellular metabolism by protein lysine acetylation. Sci. 2010;327:1000-4.

23. Kong $X$, van Diepeningen $A D$, van der Lee TAJ, Waalwijk $C$, Xu J, Xu J, Zhang $\mathrm{H}$, Chen W, Feng J. The Fusarium graminearum histone acetyltransferases are important for morphogenesis, DON biosynthesis, and pathogenicity. Front Microbiol. 2018:9:654. https://doi.org/10.3389/fmicb.2018.00654.

24. Catlett NL, Lee B, Yoder OC, Turgeon BG. Split-marker recombination for efficient targeted deletion of fungal genes. Fungal Genet Newsl. 2003;50:9-11.

25. Finkemeier I, Laxa M, Miguet L, Howden AJM, Sweetlove LJ. Proteins of diverse function and subcellular location are lysine acetylated in Arabidopsis. Plant Physiol. 2011;155:1779-90.

26. Yu F, Gu Q, Yun Y, Yin Y, Xu JR, Shim WB, Ma Z. The TOR signaling pathway regulates vegetative development and virulence in Fusarium graminearum. New Phytol. 2014;203:219-32.

27. Hu W, Zhang X, Chen X, Zheng J, Yin Y, Ma Z. a1-tubulin FaTuA1 plays crucial roles in vegetative growth and conidiation in Fusarium asiaticum. Res Microbiol. 2015;166 (3:132-42.

28. Son $H$, Seo $Y-S$, Min K, Park AR, Lee J, Jin JM, Lin Y, Cao P, Hong SY, Kim EK, Lee SH, Cho A, Lee S, Kim MG, Kim Y, Kim JE, Kim JC, Choi G, Yun SH, Lim J, Kim M, Lee YH, Choi YD, Lee YW. A phenome-Based Functional Analysis of Transcription Factors in the Cereal Head Blight Fungus, Fusarium graminearum. PLoS Pathog. 2011;7(10):e1002310.

29. Kim H-K, Jo S-M, Kim G-Y, Kim D-W, Kim Y-K, Yun S-H. A large-scale functional analysis of putative target genes of mating-type loci provides insight into the regulation of sexual development of the cereal pathogen Fusarium graminearum. PLoS Genet. 2015;11(9):e1005486.

30. Qin J, Wang G, Jiang C, Xu JR, Wang C. Fgk3 glycogen synthase kinase is important for development, pathogenesis, and stress responses in Fusarium graminearum. Sci Rep. 2015;5:8504.

31. Li C, Zhang Y, Wang H, Chen L, Zhang J, Sun M, Xu JR, Wang C. The PKR regulatory subunit of PKA is involved in regulating growth, sexual and asexual development, and pathogenesis in Fusarium graminearum. Mol Plant Pathol. 2018;19:909-21.

32. Lee Y, Son H, Shin JY, Choi GJ, Lee YW. Genome-wide functional characterization of putative peroxidases in the head blight fungus Fusarium graminearum. Mol Plant Pathol. 2018;19(3):715-30.

33. Zhang L, Li B, Zhang $Y$, Jia X, Zhou M. Hexokinase plays a critical role in deoxynivalenol (DON) production and fungal development in Fusarium graminearum. Mol Plant Pathol. 2016;17(1):16-28.

34. Jiang C, Zhang S, Zhang Q, Tao Y, Wang C, Xu JR. FgSKN7 and FgATF1 have overlapping functions in ascosporogenesis, pathogenesis and stress responses in Fusarium graminearum. Environ Microbiol. 2015;17:1245-60.

35. Zheng Z, Liu X, Li B, Cai Y, Zhu Y, Zhou M. Myosins FaMyo2B and Famyo2 affect asexual and sexual development, reduces pathogenicity, and FaMyo2B acts jointly with the myosin passenger protein FaSmy1 to affect resistance to Phenamacril in Fusarium asiaticum. PLoS One. 2016;11(4): e0154058. 
36. Yin $Y$, Wang Z, Cheng D, Chen X, Chen Y, Ma Z. The ATP-binding protein FgArb1 is essential for penetration, infectious and normal growth of Fusarium graminearum. New Phytol. 2018;219(4):1447-66.

37. Josefsen L, Droce A, Sondergaard TE, Sørensen JL, Bormann J, Schäfer W, Giese H, Olsson S. Autophagy provides nutrients for nonassimilating fungal structures and is necessary for plant colonization but not for infection in the necrotrophic plant pathogen Fusarium graminearum. Autophagy. 2012;8(3):326-37.

38. Liu H, Zhang S, Ma J, Dai Y, Li C, Lyu X, Wang C, Xu JR. Two Cdc2 kinase genes with distinct functions in vegetative and infectious hyphae in Fusarium graminearum. PLoS Pathog. 2015;11:e1004913.

39. Alexander NJ, McCormick SP, Larson TM, Jurgenson JE. Expression of Tri15 in Fusarium porotrichioides. Curr Genet. 2004;45:157-62.

40. Wu X, Vellaichamy A, Wang DP, Zamdborg L, Kelleher NL, Huber SC, Zhao YF. Differential lysine acetylation profiles of Erwinia amylovora strains revealed by proteomics. J Proteome. 2013;79:60-71.

41. Shannon P, Markiel A, Ozier O, Baliga NS, Wang JT, Ramage D, Amin N, Schwikowski B, Ideker T. Cytoscape: a sofware environment for integrated models of biomolecular interaction networks. Genome Res. 2003;13:2498-504.

42. Lin YW, Beharry ZM, Hill EG, Song JH, Wang W, Xia Z, Zhang Z, Aplan PD, Aster JC, Smith CD, Kraft AS. A small molecule inhibitor of Pim protein kinases blocks the growth of precursor T-cell lymphoblastic leukemia/ lymphoma. Blood. 2010;115(4):824-33.

43. Beharry Z, Mahajan S, Zemskova M, Lin YW, Tholanikunnel BG, Xia Z, Smith CD, Kraft AS. The Pim protein kinases regulate energy metabolism and cell growth. Proc Natl Acad Sci U S A. 2011;108(2):528-33.

44. Jimeno-Gonzalez S, Gomez-Herreros F, Alepuz PM, Chavez S. A gene-specific requirement for $\mathrm{FACT}$ during transcription is related to the chromatin organization of the transcribed region. Mol Cell Biol. 2006;26:8710-21.

45. Chen Y, Wang J, Yang N, Wen Z, Sun X, Chai Y, Ma Z. Wheat microbiome bacteria can reduce virulence of a plant pathogenic fungus by altering histone acetylation. Nat Commnu. 2018;9:3429.

46. Bormann J, Boenisch MJ, Bruckner E, Firat D, Schafer W. The adenylyl cyclase plays a regulatory role in the morphogenetic switch from vegetative to pathogenic lifestyle of Fusarium graminearum on wheat. PLoS One. 2014;9:e91135.

47. Hu S, Zhou X, Gu X, Cao S, Wang C, Xu JR. The cAMP-PKA pathway regulates growth, sexual and asexual differentiation, and pathogenesis in Fusarium graminearum. Mol Plant-Microbe Interact. 2014;27(6):557-66.

48. Peplow AW, Tag AG, Garifullina GF, Beremand MN. Identification of new genes positively regulated by Tri10 and a regulatory network for trichothecene mycotoxin production. Appl Environ Microbiol. 2003;69(5):2731-6.

49. Zhou XY, Heyer C, Choi YE, Mehrabi R, Xu JR. The CID1 cyclin C-like gene is important for plant infection in Fusarium graminearum. Fungal Genet Biol. 2010;47:143-51.

50. Jiang C, Zhang C, Wu C, Sun P, Hou R, Liu H, Wang C, Xu JR. TRI6 and TRI10 play different roles in the regulation of deoxynivalenol (DON) production by CAMP signalling in Fusarium graminearum. Environ Microbiol. 2016;1 1:3689-701.

51. Yin T, Zhang Q, Wang J, Liu H, Wang C, Xu JR, Jiang C. The cyclaseassociated protein FgCap1 has both protein kinase A-dependent and -independent functions during deoxynivalenol production and plant infection in Fusarium graminearum. Mol Plant Pathol. 2018;19(3):552-63.

52. Li D, Lv B, Tan L, Yang $Q$, Liang W. Acetylome analysis reveals the involvement of lysine acetylation in diverse biological processes in Phytophthora sojae. Sci Rep. 2016;6:29897.

53. Xiong Y, Peng X, Cheng Z, Liu W, Wang GL. A comprehensive catalog of the lysine-acetylation targets in rice (Oryza Sativa) based on proteomic analyses. J Proteome. 2016;138:20-9.

54. Petersen B, Petersen TN, Andersen P, Nielsen M, Lundegaard C. A generic method for assignment of reliability scores applied to solvent accessibility predictions. BMC Struct Biol. 2009;9:51.

55. Kanehisa M, Goto S, Kawashima S, Okuno Y, Hattori M. The KEGG resource for deciphering the genome. Nucleic Acids Res. 2004;32:D277-80.

56. Jiao X, Sherman BT, Huang DW, Stephens R, Baseler MW, Lane HC, Lempick RA. DAVID-WS: a stateful web service to facilitate gene/protein list analysis. Bioinformatics. 2012;28:1805-6.

57. Benjamini Y, Yekutieli D. The control of the false discovery rate in multiple testing under denpendency. Ann Stat. 2001;29:1165-88.

\section{Publisher's Note}

Springer Nature remains neutral with regard to jurisdictional claims in published maps and institutional affiliations.

Ready to submit your research? Choose BMC and benefit from:

- fast, convenient online submission

- thorough peer review by experienced researchers in your field

- rapid publication on acceptance

- support for research data, including large and complex data types

- gold Open Access which fosters wider collaboration and increased citations

- maximum visibility for your research: over $100 \mathrm{M}$ website views per year

At BMC, research is always in progress.

Learn more biomedcentral.com/submissions 